\title{
General Chiral $s U_{2} \times S U_{2}$ Lagrangian and Representation Mixing.
}

A. EBraHim and F. GÜrSEY

Physics Department, Middle East Technical University - Ankara

(Lett. Nuovo Cimento, 9, 9 (1974))

1) Equation (1) should read

$$
\mathscr{L}_{\tau \psi}=-\bar{\psi} \gamma_{\mu} \partial_{\mu} \psi-m \bar{\psi} U \psi+\ldots
$$

2) The last term in eq. (5) should read

$$
-i f^{2}\left(1+g^{\prime}\right) \bar{N} \gamma_{5} \gamma_{\mu} \tau \cdot \boldsymbol{X}_{5 \mu} N
$$

3) The last term in eq. (7) should read

$$
-\frac{1}{2} m_{\varrho}^{2}\left(\rho_{\mu}+\frac{2 f^{2}}{g} \boldsymbol{X}_{\mu}\right)^{2}
$$

4) Equation (16) should read

$$
V_{\mu}^{\alpha}=\frac{1}{4 f^{2}} \partial_{\mu} u^{\alpha} \quad \text { with } u^{4}=\sigma, u^{i}=2 f \varrho \varphi^{i} .
$$

5) Equation (19) should read

$$
\exp \left[i \theta \int\left(\varphi^{2}+\pi_{0}^{2}\right) \mathrm{d}^{3} x\right] \varphi \exp \left[-i \theta \int\left(\varphi^{2}+\pi_{0}^{2}\right) \mathrm{d}^{3} x\right]=\pi_{0} \quad \text { with } \theta=45^{\circ} .
$$

6) Footnote (11) should read: Alternatively, the factor $2(1+\sigma)^{-1}$ can be tranformed away by defining a new nucleon field according to $B=\sqrt{2}(1+\sigma)^{-1} N$ as will be discussed elsewhere.

(C) by Società Italiana di Fisica

Proprietà letteraria riservata

Direttore responsabile: CARLO CASTAGNOLI

Stampato in Bologna dalla Tipografla Compositori coi tipi della Tipografla Monograf

Questo fascicolo è stato licenziato dai torchi il 23-1V-1974 